Ann. Sci. forest., 1980, 37 (4), 361-370.

\title{
Injection de solutions nutritives dans les hêtres menacés
}

\author{
Dr. Ir. R. VELDEMAN
}

Station de Phytopathologie, Gand (Belgique)

96, Burg. V. Gausberghelann, B 9220 Merelbeke

\section{Résumé \\ Injection de solutions nutritives dans les hêtres menacés}

Les conditions climatiques extrêmes de 1975-1976 ont abouti à l'affaiblissement ou à la mort de nombreux hêtres. Les hêtres sont très sensibles à l'asphyxie racinaire causée par l'engorgement des sols au printemps 1975, et n'ont pas pu se rétablir lors de l'été chaud et sec de 1976. Un système racinaire réduit n'assure plus la nutrition physiologique normale, et les mycorhizes sont endommagées ou altérées.

L'intervention la plus directe consiste à injecter des solutions dans les vaisseaux de l'arbre, utilisant une technique identique à celle employée pour lutter contre la maladie hollandaise de l'Orme.

On fournit en même femps, des éléments organiques (hydrates de carbone, acides aminés), des éléments minéraux $(N, P, K)$, des hormones et des vifamines. L'injection à la base du tronc assure une bonne pénétration dans les racines stimulant la croissance de jeunes racines ef le développement de mycorhizes.

La culture in vitro et l'inoculation dans le sol de souches mycorhiziennes issues de hêtres sains sont en cours. On a ainsi obtenu la survie de deux mille hêtres environ.

\section{Introduction et diagnostic}

La mortalité parmi les hêtres a provoqué ces dernières années une inquiétude justifiée tant parmi les propriétaires que dans l'opinion publique. D'autant plus que pendant les dernières saisons de croissance, à côté de nombreux arbres morts, un grand nombre de hêtres présentaient un feuillage réduit et avaient développé une cime peu fournie.

Ces phénomènes se présentent principalement dans le Nord de la Belgique généralement dans les drèves et les parcs.

Devenu en outre un phénomène couvrant l'Europe occidentale, la mortalité des hêtres est l'objet d'une inquiétude générale.

Comme les cas peuvent se présenter aussi bien parmi les jeunes que parmi les vieux arbres, éventuellement affaiblis, et comme la répartition est générale il faut rechercher logiquement les causes dans des troubles physiologiques dans le processus de croissance.

Vu l'urgence du problème, des recherches furent entamées à la Rijksstation voor Plantenziekten, C. L. O. Gand, pour en déterminer la portée exacle et éventuellement mettre au point des traitements de lutte. 
Incontestablement les circonstances climatiques exceptionnelles de 1975 et 1976 et leur influence sur la croissance des arbres sont en liaison avec ces phénomènes. Le niveau élevé atteint par la nappe phréatique pendant une très longue période au printemps 1975, a provoqué la mort de certaines racines par asphyxie ce qui, dans beaucoup de cas, aboutissait à la mort de l'arbre.

De nombreux hêtres se sont rétablis partiellement en subsistant sur les réserves des années précédentes, cependant cette même année une quantité suffisante de réserves ne put être constituée.

La sécheresse exceptionnelle de 1976 asséna aux arbres dépérissants un nouveau coup car ceux-ci ne purent restaurer leur système radiculaire et durent puiser dans leurs dernières réserves.

Un débourrement très défectueux en 1977, associé à un développement foliaire insuffisant constituaient les symptômes d'épuisement total dû à deux saisons successives au cours desquelles aucun rétablissement n'avait été possible. L'apparition de nécroses de l'écorce provoquée par le champignon Nectria coccinea prend alors en maints endroits des aspects inquiéfants tandis que l'Armillaria mellea se manifeste clairement sur beaucoup d'arbres affaiblis. La présence de ces parasites ne peut cependant pas être généralisée et on ne peut donc pas leur attribuer l'intégralité des hêtres morts.

\section{Analyse de la situation}

Déjà en 1975 nous avons pu observer un grand nombre de racines mortes aux différents endroits où des hêtres malades avaient été repérés. Ces constatations ont été confirmées en 1976 et 1977 . Quoiqu'il soit difficile d'interpréter quantitativement cette forme de dégât, il est indisculable que ce phénomène peuł provoquer un trouble dans le processus d'absorption de l'eau et de substances nutritives du sol. En effet, non seulement une partie importante du système radiculaire est éliminée mais la formation de nouvelles radicelles actives est exclue.

Le fait que le système radiculaire ne remplisse plus sa fonction conduit à une situation où la teneur en eau est extrêmement basse dans les structures xylémiques du tronc des tiges et des racines.

Une importante constatation sur les racines est le manque de mycorhizes, champignons qui sont particulièrement importants chez le hêtre. C'est ainsi qu'en présence de mycorhizes l'absorption du phosphore parait être cinq fois, celle de l'azote trois fois et celle de la potasse presque dix fois plus grandes.

Dans des circonstances particulières, ce facteur peut acquérir une importance telle que l'insuffisance caractérisée de ces éléments dans la sève doit être mise en corrélation avec l'absence des mycorhizes. Cette hypothèse est d'autant plus plausible que des analyses sur la présence d'éléments nutritifs dans le sol, réalisées à une dizaine d'endroits, laissaient apparaître clairement des valeurs critiques pour les éléments principaux (N, P, K).

Ces troubles physiologiques de l'arbre ont limité de manière sensible la capacité de croissance du hêtre, ce qui apparaît dans les très petifs cernes annuels formés depuis 1975. Dans la plupart des centaines d'échantillons mesurés, les accroissements radiaux de 4 à $5 \mathrm{~mm}$ étaient réduits à 1 à $2 \mathrm{~mm}$ depuis 1975. De plus, ceci signifie 
une réduction sensible du système vasculaire actif et une entrave à la circulation de la sève. Pour la sève descendante ceci sera d'autant plus crucial que la largeur des structures phloémiques n'atteint généralement que 10 p. 100 de celle des structures xylémiques. Ce phénomène est généralement un obstacle sérieux de la reconstitution spontanée du hêtre. Pour estimer la présence de réserves dans des arbres malades et sains, quelques analyses ont été faites à la méthode de Hassid ; elles nous ont fourni une base plus sûre pour apprécier l'évolution du problème. Malgré la nécessité d'autres analyses pour confirmer, on pourra retenir des tableaux $1,2,3$, des indications importantes.

\section{TABLEAU 1}

Comparaison de la présence des sucres dans l'écorce et le bois d'un hêtre et d'un peuplier (janvier 1977) Comparison of sugar contents in the bark and wood of a beech and a poplar (1977 January)

\begin{tabular}{|c|c|c|c|}
\hline & Total sucres red. & Sucres red. & Sucrose \\
\hline 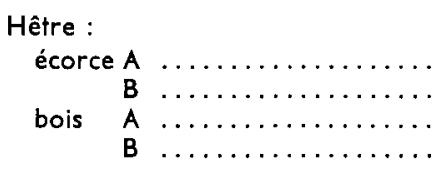 & $\begin{array}{l}4,67 \\
4,20 \\
1,73 \\
1,53\end{array}$ & $\begin{array}{l}2,43 \\
2,55 \\
1,47 \\
1,28\end{array}$ & $\begin{array}{l}2,24 \\
1,65 \\
0,26 \\
0,25\end{array}$ \\
\hline $\begin{array}{l}\text { Peuplier : } \\
\text { écorce } \quad \ldots \ldots \ldots \ldots \ldots \ldots \ldots \ldots \ldots \\
\text { bois } \ldots \ldots \ldots \ldots \ldots \ldots \ldots\end{array}$ & $\begin{array}{l}4,34 \\
1,55\end{array}$ & $\begin{array}{l}2,55 \\
1,52\end{array}$ & $\begin{array}{l}1,79 \\
0,03\end{array}$ \\
\hline
\end{tabular}

Pour les arbres sains, aussi bien chez le hêtre que chez le peuplier, une réserve en hydrates carbonnés permet la reprise de croissance au printemps.

\section{TABLEAU 2}

Répartition des sucres dans l'écorce, le bois et les racines du hêtre Assesment of sugars in bark, wood, and roots of beech

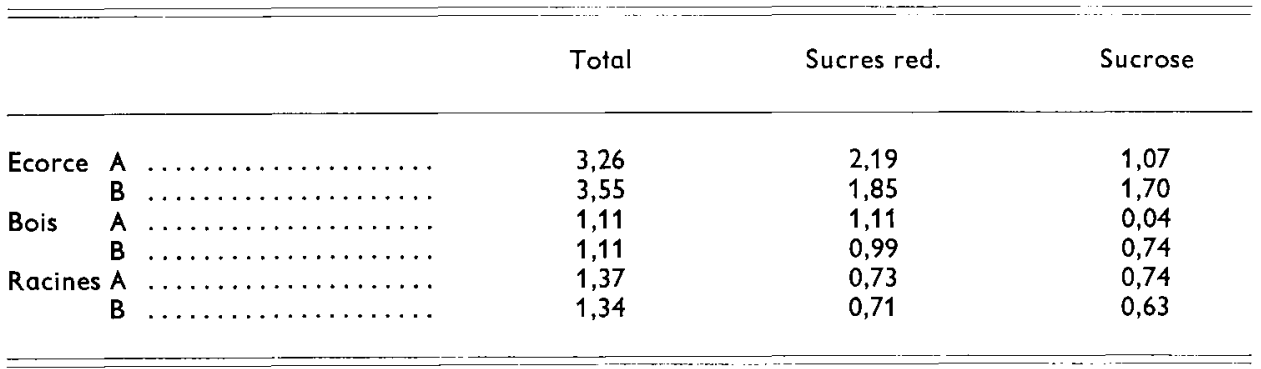

Ces données indiquent le taux de sucres dans différentes parties de l'arbre. Dans les racines les hydrates de carbone ne sont pas accumulés mais immédiatement consommés pour la formation de racines. 
TABLEAU 3

Répartition des sucres ò différentes profondeurs dans l'arbre (par $3 \mathrm{~mm}$ )

Assesment of sugars of different depth in the wood

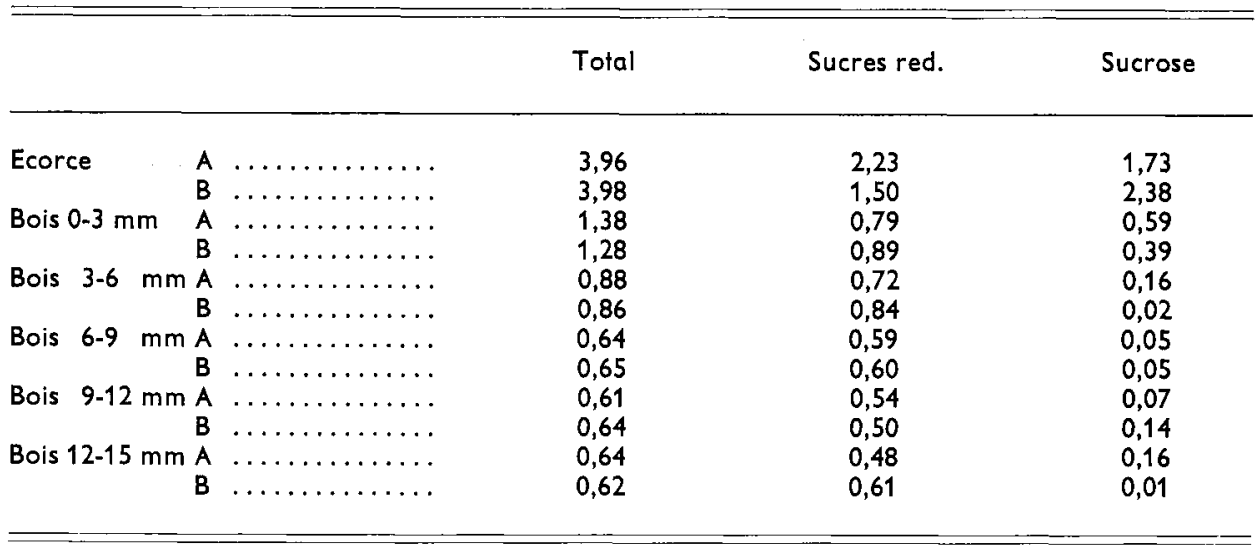

Les chiffres révèlent bien la faible teneur en réserve une fois dépassé $6 \mathrm{~mm}$. Il en résulte que seulement les cernes de croissance des dernières années peuvent intervenir comme source en sucres.

\section{Traitement}

Le désastre qui frappe actuellement les hêtres est si important qu'il nécessite des recherches sur les remèdes à appliquer. Le problème est cependant tellement urgent qu'on ne peut pas attendre les résultats de plusieurs années de recherches. II est impensable, à une époque où la conservation de la nature ne souffre pas de délai, de laisser la nature à elle-même sous prétexte que le hêtre n'est pas en station dans notre région. En se basant, d'une part, sur les connaissances sur la physiologie de l'arbre et, d'autre part, sur une analyse précise des phénomènes, il est possible d'intervenir à bon escient afin d'aider un grand nombre d'arbres à sortir de leur situation critique, ce qu'ils ne peuvent faire spontanément. Nous avons pris l'initiative, quoique à une échelle réduite, de traiter quelques milliers d'arbres. Cette initiative fut encouragée par de nombreux scientifiques qui, grâce à leurs indications précises, permirent de mettre au point la méthode de traitement.

L'intervention la plus efficiente pour aider un arbre à récupérer le plus rapidement possible est l'injection de solutions nutritives composées. En plus, il convient également d'améliorer la situation nutritive du sol et de prendre des mesures afin de favoriser le rétablissement de la flore «mycorhizienne».

\section{L'injection de solutions nutritives}

L'injection de solutions nutritives sous pression dans le système vasculaire de l'arbre a atteint ces dernières années le stade de l'application pratique. La menace de la disparition des ormes a contribué dans plusieurs pays à la généralisation tant des 
fongicides adéquats que du matériel nécessaire. Le même matériel que nous avons depuis adapté au traitement d'arbres en série, nous permit d'exécuter les premières applications expérimentales d'alimentation artificielle sur une centaine de hêtres dès mai 1977. La solution utilisée était composée principalement de divers sucres ef d'hormones de croissance des racines.

Le flux naturel de la sève est dominé par la pression de 4 à 5 atm. à laquelle est injectée la solution. Des injections effectuées à la base de l'arbre, de préférence aux ailes des racines, assurent une bonne pénétration vers celles-ci, même en période de sève ascendante. La solution fut complétée d'éléments nutritifs minéraux contenant un bon équilibre (proportion $N, P, K$ ) et à mesure que les analyses fournissaient de nouvelles données les solutions nutritives étaient adaptées. C'est ainsi que la dose de potassium était augmentée dans le cas où une carence de cet élément était détectée el que des vitamines (thiamine) étaient ajoutées afin de fournir aux mycorhizes cette substance qui leur est indispensable.

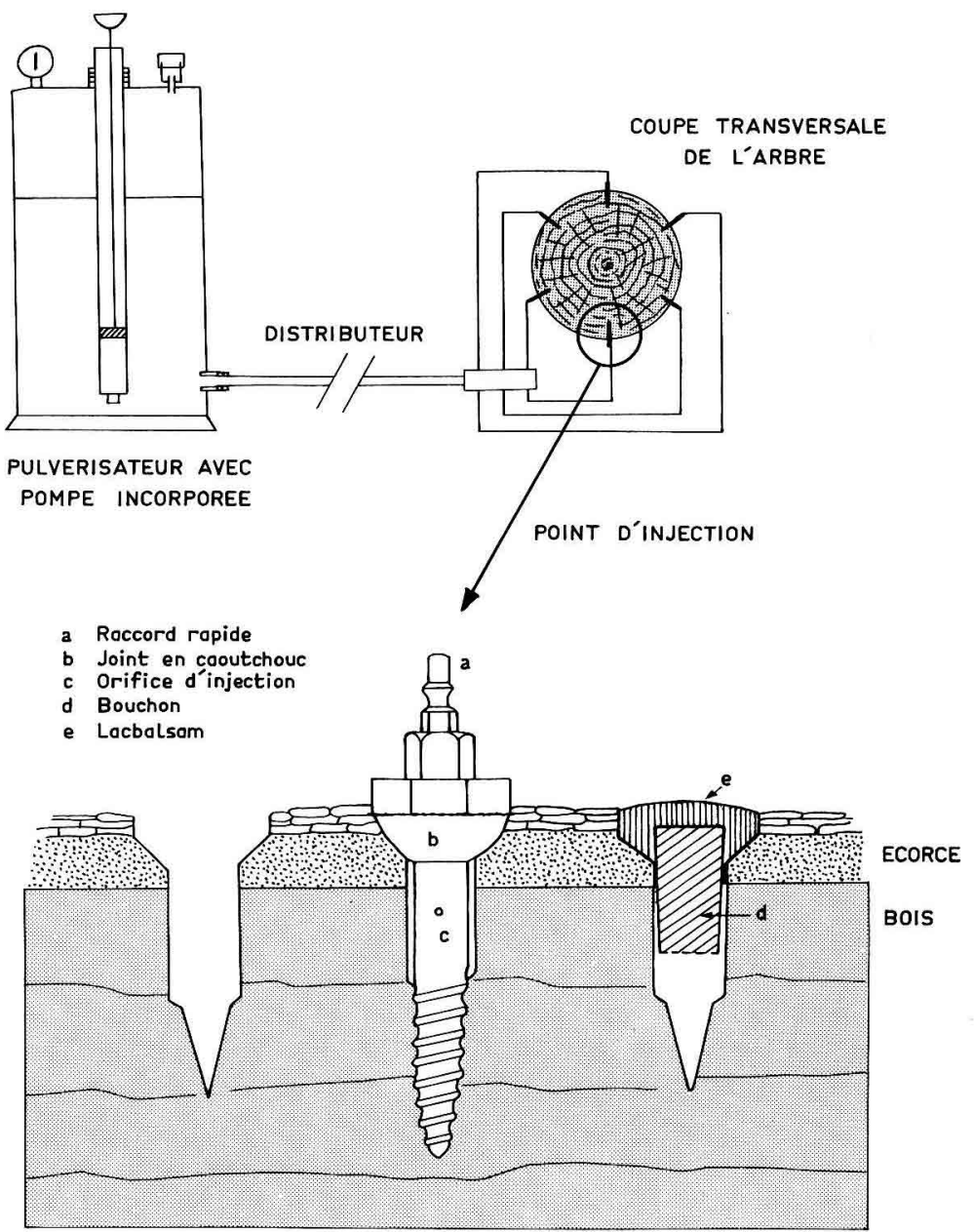

FIG. 1. 
La solution nutritive était finalement composée sur le plan organique :

4 p. 100 de sucrose,

1 p. 100 de glucose,

200 ppm acides aminés - glutamine,

$50 \mathrm{ppm}$ thiamine chlorate,

$$
\text { - asparagine, }
$$

5 ppm d'acide indol butyrique.

Pour les minéraux nous avons fait usage de composés liquides à différents taux d'azote, de phosphore et de potasse, à des concentrations de $1 \mathrm{~g} / \mathrm{l}$. Un engrais composé dans le rapport 5-7-10 nous paraît finalement le plus indiqué.

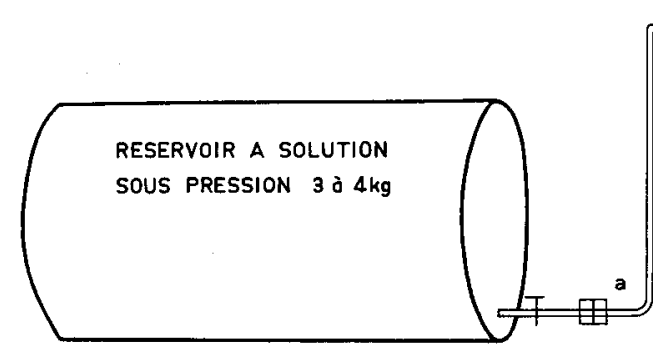

REMPLISSAGE DU BIDON DE SOLUTION
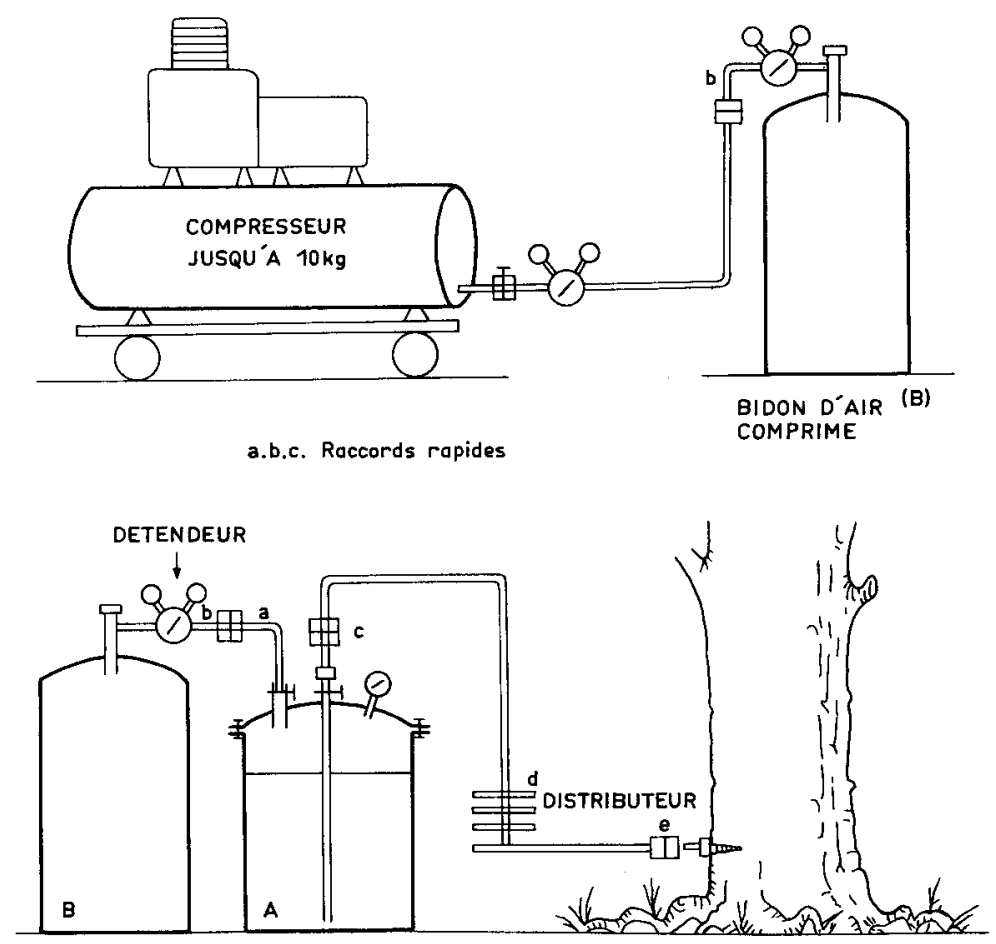

FIG. 2. 
Comme pour le traitement des ormes la quantité injectée fuł déterminée en fonction de la grandeur de l'arbre à traiter, c'est-à-dire en injectant 11 de solution par $30 \mathrm{~cm}$ de circonférence.

Dans le cas d'arbres exceptionnels, l'injection fut répétée et le volume de solution injectée fortement augmenté. Le traitement fut exécuté pendant toute l'année, de préférence pendant une période de croissance active et sauf pendant la période de risque de gelée.

La technique d'injection est illustrée par les figures 1 et 2 ef la photo 1 . Des trous sont forés à la base du tronc au moyen d'une tarière profilée. Les chevilles d'injection sont introduites et serrées dans les trous, jusqu'à atteindre une bonne étanchéité entre le joint et le collet dans l'écorce. Les conduites sont fixées sur les chevilles au moyen de raccords rapides. Quand l'opération est terminée, les chevilles sont dévissées et le trou est fermé par un bouchon bien adapté, le tout étant recouvert avec un goudron protecteur adéquat. La solution est injectée à une pression de 4 à $5 \mathrm{~kg}$. De cette manière, on a compensé le rôle du feuillage partiellement manquant et les racines ont eu à leur disposition une bonne dose d'énergie qui, à son tour, fut rapidement utilisée pour une croissance active.

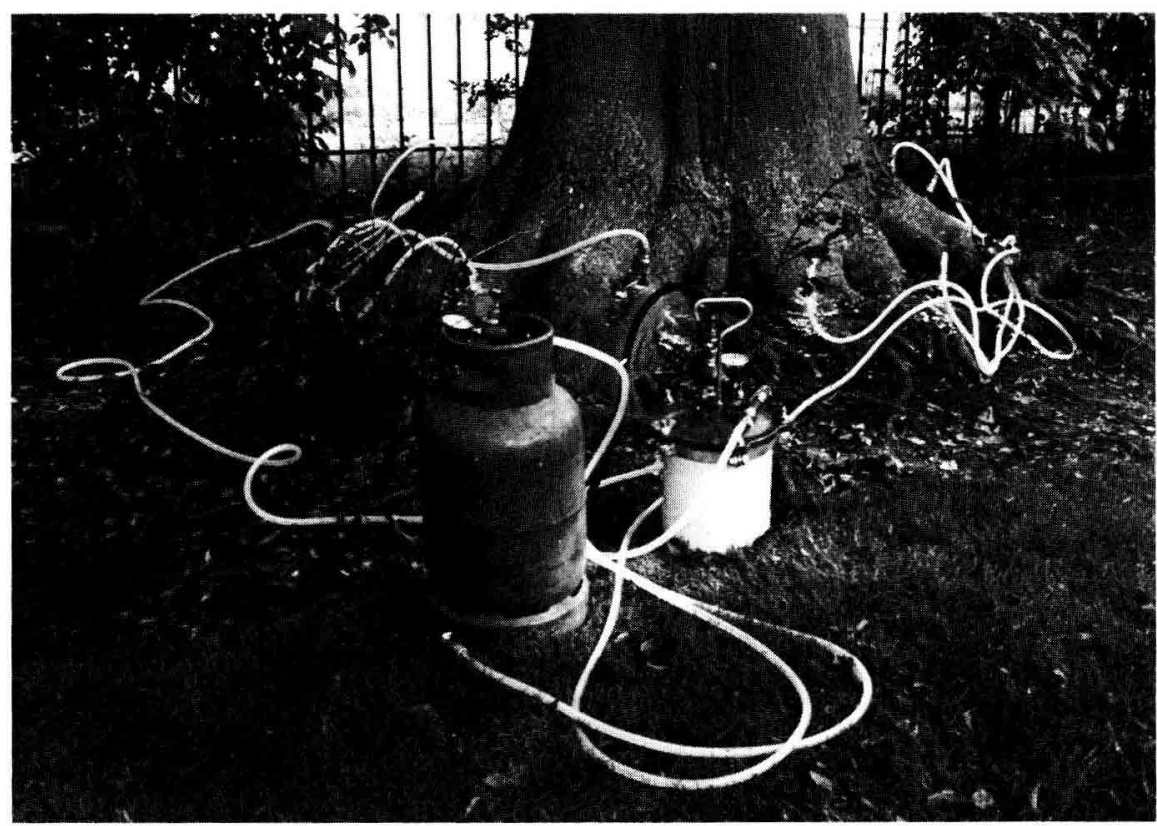

PHOTO 1. - Injection de solutions nutritives dans les racines d'un hêtre.

A la fin de la période de végétation, plusieurs centaines d'arbres furent encore approvisionnés de la quantité nécessaire de substances de réserves nécessaires à la formation de racines pendant l'arrière saison el au débourrement de bourgeons et la formation de feuilles au printemps. 


\section{Inoculation de mycorhizes dans le sol}

Le hêtre semble extrêmement sensible à la présence des mycorhizes sur les racines. Plus encore que les racines, les mycorhizes ont été fortement endommagés par les situations édaphiques défavorables les années 1975-76. Les effets bénéfiques des mycorhizes sur la plante se révèlent surtout dans la nutrition minérale ainsi que sur la répartition de la biomasse dans la cime et les racines (rapport tige/ racine).

Le développement des mycorhizes sur les racines est déterminé, d'une part, par une nutrition provenant de la plante hôte (hydrates de carbone et vitamines), mais, d'autre part, aussi bien par les conditions de croissance (aération - conditions chimiques du sol).

L'injection de la solution nutritive dans l'arbre peut fournir l'énergie nécessaire pour favoriser le développement des mycorhizes, car une grande partie de la solution descend vers les racines. L'addition de vitamines (thiamine) aux solutions injectées est surtout destinée aux mycorhizes, car pour ce groupe de champignons ces éléments sont indispensables. Ceux-ci sont normalement formés dans les feuilles, puis descendent vers les racines. Une feuillaison insuffisante entraîna l'affaiblissement de la flore mycorhyzienne et même sa disparition.

Pour inoculer les racines dans le cas où les mycorhizes étaient perdues, les premiers essais ont commencés. Les mycorhizes des racines de hêtres forestiers sains sont cultivées in vitro sur les substrats adéquats, puis multipliées par inoculation dans un mélange organique enrichi.

Ainsi, déjà cinq champignons (des basidiomycètes), jusqu'à maintenant indéterminés, ont été cultivés. Ces cultures, mélangées avec des granules d'argex, sont enfouies dans des trous de $10 \mathrm{~cm}$ de diamètre, à de multiples endroits dans la zone des racines. Cette opération favorise en même temps aussi bien l'aération du sol, effet qui se traduit en une formation de racines nouvelles.

\section{Résultats}

Au cours de la période de 2 ans d'expérimentation, plus de deux mille hêtres ont été traités. Principalement pendant l'année 1977, des centaines d'arbres répartis dans le Nord de la Belgique ont bénéficié d'une injection. Alors que des milliers d'arbres souffraient, seuls les plus menacés ont été choisis pour le traitement, faute de pouvoir tous les traiter.

Généralement, l'intervention a été bénéfique, assurant la survie de l'arbre et une reprise supérieure à celle des arbres non traités. Des contrôles effectués sur les racines ont démontré la naissance de radicelles souvent bien mycorhizées, effet difficile à chiffrer.

L'effet sur la formation de réserves en sucres est illustré dans le tableau 4. 


\section{TABLEAU 4}

Teneur en sucres dans l'écorce ef dans les racines de hêtres sains, malades et traités Sugar contents in the bark and the roots respectively of healthy, diseased, and treated beeches

\begin{tabular}{|c|c|c|c|c|c|c|c|}
\hline & & \multicolumn{2}{|c|}{ Total } & \multicolumn{2}{|c|}{ Sucres red. } & \multicolumn{2}{|c|}{ Sucrose } \\
\hline & & écorce & racines & écorce & racines & écorce & racines \\
\hline $\begin{array}{l}\text { Sains } \\
\text { Malades } \\
\text { Traités }\end{array}$ & 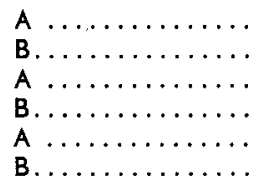 & $\begin{array}{l}3,33 \\
3,00 \\
1,43 \\
1,33 \\
5,61 \\
4,61\end{array}$ & $\begin{array}{l}0,84 \\
0,85 \\
0,73 \\
0,50 \\
1,37 \\
1,44\end{array}$ & $\begin{array}{l}1,85 \\
1,75 \\
1,02 \\
0,99 \\
1,76 \\
1,70\end{array}$ & $\begin{array}{l}0,76 \\
0,64 \\
0,74 \\
0,60 \\
1,80 \\
1,12\end{array}$ & $\begin{array}{l}1,48 \\
1,25 \\
0,41 \\
0,34 \\
3,85 \\
2,91\end{array}$ & $\begin{array}{l}0,10 \\
0,20 \\
- \\
\overline{0,37} \\
0,32\end{array}$ \\
\hline
\end{tabular}

II en résulte que non seulement l'écorce mais aussi bien les racines sont bien pourvues en hydrates de carbone provenant de l'injection. Quelques arbres dans un état critique, mais de valeur exceptionnelle, traités à plusieurs reprises se sont rétablis et pourront donner lieu à une régénération naturelle.

\section{Discussion}

En intervenant dans le cycle de nutrition organique de la plante par injection sous pression dans les tissus vasculaires, on découvre de nombreuses possibilités qui ne sont pas encore toutes connues. Bien que jusqu'à ce jour appliquées seulement pour l'endothérapie et pour remédier à certaines carences, les techniques d'injection peuvent offrir des possibilités nouvelles d'intervention au niveau des racines.

Dans le cas du hêtre que nous avons étudié, la formation des racines et des mycorhizes a été stimulée. Avec des fongicides convenables, la lutte contre l'Armillaria devient possible. Si les techniques d'injection sont bien connues, il reste beaucoup à faire dans le domaine des solutions à employer.

Reçu pour publication en décembre 1979.

\section{Summary}

\section{Intervention by artificial injection of nutrient solutions to menaced beeches}

The last season a lot of beeches have suffered or died by insufficiencies owed to the extreme weather conditions in 1975-1976. Indeed, several thousand of beeches, old and young ones, are already dead, or have a poor growth. Beeches are very susceptible for root asphyxiation caused by the high water level in the soil in spring 1975 so that several trees died already that year, other ones could not be reestablished during the following hot and dry growing season.

Reduced root systems could not ensure the normal physiological processes, wile also the mycorrhizes are dammaged or killed.

The most direct intervention in the nutrient supplies of the tree can be obtained by injection of solutions into the transporisystems.

injection technics for the application of fungicides are already common for control of Dutch elm disease. The same technics were used to inject nutrient solutions in beeches. 
At the same time, organic elements (carbonhydrates-amino acids) anorganic elements (N, P, K) hormons and vitamins were given. Injection at the base of the tree ensured a good penetration in the roots, so that the formation of young roots, and the development of mycorrhizes was stimulated.

Cultivation in vitro of some isolates from healthy beeches in forest, is studied, and already inoculation of the soil with inoculated substrates has been applied.

Good results for the survical of about two thousand trees have been obtained.

\section{Zusammenfassung}

\section{Einspritzung von Nährstoffe in die erkrankte Büche}

Durch die schlechte Wetterbedingungen von 1975 und 1976, sind viele Buchen erkrankt oder sogar schon abgestorben. Weil Buchen sehr anfällig sind for erstickung durch sauerstoffmangel, sind durch den hohen grundwasserspiegel vom Frühjahr $1975 \mathrm{im}$ gleichen jahr schon viele abgestorben. Erkrankte Baüme konnten in die darauffolgende Wachtstumperiode nicht regenerieren, wegen der aussergewöhnlichen Hitze und Trockenheit.

Wegen der Würzelbeschädigung, war eine völlige Physiologische Entwickelung nicht mehr möglich, es sind ausserdem viel Mykorrhizen abgestorben oder beschädigd.

Der wieksamste Eingriff im Ernährungsprozess bein diesem Baümen isł die directe Einspritzung von Nährstoffen im safttransportsystem.

Injectiontechniken sind schon algemein angewendet in der Ulmkrankheitbekämpfung. Ahnliche Techniken verwenden wir bei der Nährstoffgabe.

Es werden gleichzeitig organische und anorganische Elemente, Hormone und Vitaminen zugeführt. Injectionen im Würzelansatz des Baumes versicheren eine gute Penetration in die Tiefe. So bilden sich junge Würzeln, und eine gute Mykorrhizenentwickelung is gewährleistet.

Aus gesunde würden Waldbuchen Mykorrhizen isoliert und in Vitrokulturen vermehrfacht. Anschliessend werden diese auf substraten inocculiert und in die Wurzelzone des erkrankten Baumes introduziert.

2000 Baüme würden mit gutem Erfolg auf dieser Weise behandelt. 